Note. This is an accepted manuscript version for an article to be published in the journal Food Quality and Preference; the current version might differ slightly from the published version.

\title{
People Overestimate Verbal Quantities of Nutrients on Nutrition Labels
}

Dawn Liu ${ }^{1}$, Marie Juanchich ${ }^{1}$, Miroslav $\operatorname{Sirota}^{1}, \&$ Sheina Orbell ${ }^{1}$

${ }^{1}$ Department of Psychology, University of Essex, Wivenhoe Park, Colchester, CO4 3SQ, United Kingdom

Correspondence regarding this article should be addressed to the first author at dliuxi@essex.ac.uk / dawn.liu@ymail.com

Data and materials from the studies are available on the Open Science Framework at https://osf.io/yjxzd/?view_only=391dcf90246d4cf2be5a031a9fc42d34. 


\title{
INTERPRETATIONS OF NUTRIENT QUANTITIES
}

\begin{abstract}
Nutrition labels provide information about nutrient quantities in food, thus offering consumers a tool to make healthy eating choices. These labels are often presented with verbal quantity information (e.g., 'low'). However, little is known about how consumers actually interpret this information. We investigated whether participants' translations of verbal quantities fit standard guidelines, and whether nutrient valence and individual differences predicted interpretational variability. In Experiment 1, participants $(N=82)$ gave numerical percentages for five verbal quantities, selected a verbal expression that best described eight numerical quantities, and estimated the amount conveyed by quantities of both formats on a visual analogue scale (all quantities and nutrients manipulated within-subject). In Experiment 2, participants $(N=801)$ translated five verbal quantities into numerical percentages. Participants interpreted quantities with great variability (SDs for given estimates ranging from $12-30 \%$ ). About $50 \%$ of participants substantially overestimated the numerical value of verbal quantities as compared to food labelling guideline ranges. Participants' estimates were greater for minerals than fat in Experiment 2. The magnitude of estimations persisted across participants with different individual characteristics. Thus, consumers misinterpret verbal labels by overestimating the quantities they describe. It could be beneficial to refine guideline ranges for nutrient values to better match people's intuitive interpretation of verbal quantities.
\end{abstract}

Keywords: food labels; quantities; verbal-numerical formats; quantity estimates; guideline daily amounts; nutrition communication 
INTERPRETATIONS OF NUTRIENT QUANTITIES

People Overestimate Verbal Quantities of Nutrients on Nutrition Labels $1 \quad$ Introduction

From an individual and a public health perspective, the ability to judge the healthiness of food is important to combat the global increase in diet-related disease (Crockett et al., 2018). Nutrition labelling is intended to empower people to make informed and healthier choices about food consumption (Crockett et al., 2018; Hawley et al., 2013). Further, verbal descriptors were introduced to simplify and categorise numerical quantities on nutrition labels (Malam, Clegg, Kirwan, \& McGinigal, 2009). Specifically, the terms 'low', 'medium', and 'high' provide consumers with a quicker and more intuitive understanding of nutrient amounts (Malam et al., 2009). Official guidelines specify what numerical ranges verbal labels should correspond with (UK Department of Health, 2016), and when such terms (e.g., 'high in minerals') can be used in manufacturer's nutrition claims (UK Department of Health, 2011). However, these interpretational guidelines are rarely presented at point of sale. While consumers report such verbal banding to be simpler and easier to use (Shannon, 1994; Temple \& Fraser, 2014), findings on whether verbal labels result in healthier choices are inconsistent (see Hersey, Wohlgenant, Arsenault, Kosa, \& Muth, 2013, for a review). Also, researchers have yet to investigate how consumers actually interpret the verbal labels.

Three theoretical and practical issues are relevant to the interpretation of verbal quantities. First, what do verbal quantities like 'low', 'medium', and 'high' mean to consumers and how much do they vary across people and contexts? There is no direct evidence about how people interpret verbally communicated food quantities, however discrepancies in people's interpretations of verbal quantifiers have been demonstrated in domains such as medical side effects (Berry, Raynor, \& Knapp, 2003) and climate change outcome likelihoods (Budescu, Por, Broomell, \& Smithson, 2014). People 


\section{INTERPRETATIONS OF NUTRIENT QUANTITIES}

derive different interpretations of a same verbal probability depending on the context and their knowledge of the subject (Knapp, Gardner, \& Woolf, 2015; Harris, Corner, \& Hahn, 2009; Sirota \& Juanchich, 2015). This affects their perceptions of a quantity, and their subsequent decisions (Berry et al., 2003; Juanchich, Sirota, \& Butler, 2012). It is therefore important to ascertain what ranges people actually ascribe to verbal quantities such as 'low', 'medium', and 'high', rather than assuming a consistent and accurate interpretation of these terms.

Second, do people's interpretations of verbal nutrient quantities match the intended meaning postulated in standard guidelines? When official numerical translations are smaller than people's intuitive understanding of the verbal term, people are likely to overestimate verbal quantities. This is observed with verbal risk frequencies for medical side effects: the EU assigns a frequency of 1-10\% for 'common' but respondents believed it to mean $44 \%$ on average (Berry, 2006). Verbal banding for nutrition labelling likewise focuses on quantities below $50 \%$, with cut-offs for 'high' amounts around $25-30 \%$. People might thus overestimate nutrient quantities depicted by verbal labels compared to official standards. This could contribute to suboptimal decisions about consumption. In the medical literature, people who overestimate the verbal frequency of a medical side effect expected more that they would experience the side effect, which could make them more averse to treatment (Knapp, Raynor, \& Berry, 2004) or increase their likelihood of developing actual side effects (Webster, Weinman, \& Rubin, 2017a). In a nutrition context, overestimations of nutrients such as vitamins in food could lead people to expect that they consume enough vitamins when in fact their diet lacks vitamins. One could thus believe their diet is healthy despite not meeting dietary targets (Craig \& Shelton, 2008). 


\section{INTERPRETATIONS OF NUTRIENT QUANTITIES}

Finally, what factors contribute to variations in quantity interpretation? Individual differences among people can predispose them to over- or underestimating verbal quantities. For example, in the medical context, less numerate individuals display poorer understanding of verbal and numerical risk frequencies, (Gardner, McMillan, Raynor, Woolf, \& Knapp, 2011) and are less consistent in providing numerical interpretations for the same scale (Lipkus \& Peters, 2009). Individuals with lower health literacy also struggle with quantitative health decisions, although motivation to process information can compensate for lower literacy (Hibbard, Peters, Dixon, \& Tusler, 2007). In a nutrition context, individuals vary in their experience with nutrition labels and motivations for healthy eating. A person who frequently consults nutrition labels because they are motivated to eat healthily may be more familiar with industry standards in translating verbal quantities, resulting in more accurate interpretations (Gigerenzer, Hertwig, van den Broek, Fasolo, \& Katsikopoulos, 2005). Additionally, characteristics about one's worldview may influence the translation of a verbal quantifier. For instance, more optimistic individuals overestimate the frequency of 'uncommon' side effects more (Webster, Weinman, \& Rubin, 2017b). The desire to draw a certain conclusion (Piercey, 2009) or support one's beliefs (Budescu, Por, \& Broomell, 2012) could motivate interpretations: someone who values healthy eating might be more motivated to justify their choices as healthy (Rayner, Boaz, \& Higginson, 2001), and therefore translate quantities in a way that fits this motivation (e.g., reassuring themselves that a 'high \%' of a 'negative' nutrient is a lower numerical value). Finally, some nutrients are associated with poorer health outcomes than others (e.g., minerals are positive, but fat is negative; Oakes, 2005a) might influence the interpretation of their quantities. People might wish to believe desirable nutrient 


\section{INTERPRETATIONS OF NUTRIENT QUANTITIES}

quantities to be higher than undesirable ones (Kunda, 1990) and provide higher estimates for positive than negative nutrients.

The two experiments presented here aimed to understand how people interpret verbal quantities in three ways. First, we aimed to determine what were people's actual interpretations of verbal quantities, hypothesising that numerical estimates of verbal quantities would vary widely between individuals. Second, we investigated whether and to what extent these interpretations fell outside the range of recommended guidelines. Finally, we explored factors that might moderate variability in people's perceptions of nutrient quantities. To address these aims, we used translation tasks from the literature on verbal probabilities (Collins \& Hahn, 2018) to test participants' interpretations of verbal nutrition quantifiers. While this method allowed us to solicit interpretations of quantities along a fixed scale $(0-100 \%)$, it required that we standardise values of nutrients. Therefore, we expressed quantifiers in terms of the percentage of their reference intake provided by a food (or 'Guideline Daily Amount'; hereafter 'GDA'). GDA labels are the most widespread among front-of-package food labels across the EU (Storcksdieck genannt Bonsmann et al., 2010) and are most viewed when consulting nutrition labels (Grunert, Fernández-Celemín, Wills, Storcksdieck genannt Bonsmann, \& Nureeva, 2010). Although official verbal quantity ranges are determined in terms of amount per 100g, verbal quantities are often presented with GDA percentages, and we believe that consumers could interpret them accordingly.

\section{Methods}

We conducted two survey-based experiments to investigate people's interpretations of verbal GDA percentages. Informed consent was obtained from all participants prior to beginning the survey, and participants were shown a debrief page at 


\section{INTERPRETATIONS OF NUTRIENT QUANTITIES}

the end of the survey explaining the purposes of the experiment. Both studies were approved by the university's ethical review committee.

\subsection{Participants}

Participants in Experiment 1 were recruited from a volunteer list of a UK university, and participants in Experiment 2 were recruited from a survey panel of a nationwide sample. In Experiment 1, 82 out of 109 participants completed the survey ( $83 \%$ female; age range $18-66$ years, $M=21.4, S D=7.9$ ). Undergraduates in the sample were given module credits for participation; non-students participated voluntarily. The sample was 53\% White, $16 \%$ Asian, and 20\% African (11\% other races); $45 \%$ had a university degree. Participants' average estimated Body Mass Index (BMI) was 22.0 $(S D=5.3)$. They reported an average attitude towards healthy eating of 4.9 on a 7 -point scale $(S D=1.0)$, and $48 \%$ reported frequent use of nutrition labels.

In Experiment 2, we used quota sampling to determine the demographics of the sample. The purpose was to address the limitation in Experiment 1 of having a small and relatively homogeneous and well-educated sample that would have reasonable understanding of GDA labels (Grunert, Wills, \& Fernandez-Celemin, 2010) and familiarity with general nutritional guidelines (Blitstein \& Evans, 2006; Parmenter, Waller, \& Wardle, 2000). Through a survey panel, as part of an unrelated study investigating feelings about seeing clusters of holes (our questions were included at the end of the survey), we obtained data from 801 participants (52\% female; age range 18 74 years), who received shopping vouchers for participation. Four percent of invitees responded to the invite link, and $18 \%$ of eligible respondents completed the study. The selection and exclusion criteria, along with the response rate, are shown in Figure 1. Full socio-demographic characteristics for our participants are shown in Table 1. Compared to Experiment 1, there was a larger range of ages and education levels, but 
INTERPRETATIONS OF NUTRIENT QUANTITIES

reported eating attitudes and use of nutritional labels were similar $\left(M_{\text {attitudes }}=4.7, S D=\right.$ $1.1 ; 49 \%$ reported frequent use of nutritional labels). Average BMI was higher than Experiment $1(M=27.4, S D=6.7)$.

\subsection{Questionnaire Development}

We developed three sets of translation tasks to address our goal of understanding people's interpretations of verbal nutrient quantities (see middle panel of Figure 2). We piloted the task questions with five volunteers to check the clarity of words and phrases used.

\subsubsection{Interpretation of verbal labels}

As our main measure of participants' interpretations of verbal quantities, we used a common method in the verbal probability translation literature (Collins \& Hahn, 2018). Participants translated five verbal labels (see Table 2) by answering the question, 'What percentage of a guideline daily amount (GDA) of [nutrient] do you think the food label describes? (Please give a number.)' Low, medium, and high were chosen as they reflect the verbal banding used in many nutrition labelling systems (Hersey, Wohlgenant, Arsenault, Kosa, \& Muth, 2013). Very low and very high were added to supplement the range of verbal descriptors and capture possible extremes of the range.

\subsubsection{Matching to numerical percentages}

To assess the consistency of participants' numerical interpretations, we solicited back-translations of numerical quantities into verbal ones by adapting a procedure previously used in translating numerical probabilities (Du \& Stevens, 2011). Participants matched eight numerical labels to verbal quantifiers. To provide their answers, they selected from a multiple-choice list of five verbal quantities (very low, low, medium, high, very high).

\subsubsection{Quantity perception}




\section{INTERPRETATIONS OF NUTRIENT QUANTITIES}

To check whether participants' interpretations of verbal and numerical quantities converged, we sought a standardised comparison between perceptions verbal and numerical quantities. We used a visual analogue measure that is commonly used in measuring pain perception, and compared against verbal and numerical rating scales (Jensen Hjermstad et al., 2011). For the 13 verbal and numerical labels, participants estimated the GDA proportion described by the specified quantity (verbal or numerical) on a visual analogue scale with three anchor points (none, half, and all) corresponding to $0-100$ with invisible increments of 1 .

\subsection{Materials and Procedure}

\subsubsection{Experiment 1}

We used a within-subject design in Experiment 1, presenting the 13 GDA labels with a range of nutrients (see Figure 2). After providing informed consent and before completing the interpretation tasks, participants completed a healthiness ranking of eight nutrients. Participants ranked a list of the randomly-ordered nutrients according to their importance in determining (1) the healthiness and (2) the unhealthiness of food. We used the selected rankings for this task to assign the verbal and numerical quantities participants would see in conjunction with a nutrient, to ensure that participants saw all nutrients and all values, but were not overwhelmed by having to rate all possible combinations. Based on the mean ranks of nutrients, we also categorised the four most important nutrients for determining healthiness as 'positive' (minerals, protein, calories, and fibre) and the four most important for determining unhealthiness as 'negative' (sugar, fat, sodium, and saturates). This provided an indication of each nutrient's relative valence.

Participants next completed the three interpretation tasks in a random order for each participant (see Figure 2). The quantities in each appeared with a different nutrient, 


\section{INTERPRETATIONS OF NUTRIENT QUANTITIES}

and the assignment of quantity to nutrient varied across participants. Within each task, the labels were presented separately (one label per page) and in a randomised order for each participant. After each task, participants rated how easy they found the task on a five-point Likert scale (1: extremely difficult, 5: extremely easy).

At the end of the survey, we also measured: (1) participants' attitudes towards healthy eating using the seven-question healthy eating motivation section of the Food Choice Questionnaire, for example, 'It is important that the food I eat keeps me healthy' (Steptoe, Pollard, \& Wardle, 1995; Cronbach's $\alpha=.78$ ); (2) frequency of nutritional label use (agreement on a seven-point scale with the statement: 'I often use nutritional labels to determine the healthiness of food.'); (3) estimated BMI: participants reported estimates of their weight $(\mathrm{kg})$ and height $(\mathrm{m})$ by selecting from a drop-down list of six weight and height ranges. Estimated BMI was calculated for each participant by taking the middle value of the weight range divided by the square of the middle value of the height range; and (4) socio-demographic characteristics (age, gender, ethnicity, and highest level of education).

\subsubsection{Experiment 2}

In Experiment 2, we aimed to test if a larger and more diverse sample would also overestimate verbal nutrient quantities. To reduce response time, we streamlined the survey to include only the verbal interpretations. Doing so also meant that participants would not be primed to consider how the labels should be translated back and forth between verbal and numerical versions. We focused on verbal-numerical translations as this allowed us to make more precise comparisons; this technique is also the most common in the literature for soliciting verbal quantity interpretations (Collins \& Hahn, 2018). We also simplified the task such that participants only translated the five verbal labels for a single nutrient. The nutrient was either minerals or fat (randomly 


\section{INTERPRETATIONS OF NUTRIENT QUANTITIES}

allocated). These were two nutrients that had been clearly categorised as healthy (minerals) or unhealthy (fat), which would allow us a better test of nutrient valence as a factor predicting interpretational variance. The between-subjects design also addressed the possibility that the within-participant design of Experiment 1 might have resulted in participants anchoring their subsequent interpretations to the first nutrient they saw.

In the individual differences section, we amended the BMI estimate measure in Experiment 1 (which took the median of participants' selected weight range) that might have limited our analysis of this variable. To get a more accurate measure, participants provided their weight in kilograms and height in centimetres. They also completed a reduced, four-question version of the attitudes towards healthy eating measure (Steptoe et al., 1995; Cronbach's $\alpha=.72$ ) and indicated their frequency of nutrition label use.

\section{$3 \quad$ Analyses}

Analyses were conducted using IBM SPSS Statistics for Macintosh, Version 24.0. To determine people's actual interpretations of verbal quantities, we analysed the numerical percentages that participants assigned to each verbal label using repeated measures ANOVAs. For the back-translations of the verbal descriptors assigned to numerical labels, we performed rank-order correlational analyses. We focused our analysis on the five numerical quantities that best matched interpretations of the five verbal quantities (see Table 2). Interpretations of the other numerical quantities $(10 \%$, $75 \%$, and $90 \%$ ) are included as supplementary materials on the Open Science Framework (https://osf.io/yjxzd/?view_only=391dcf90246d4cf2be5a031a9fc42d34).

To determine if participants' interpretations matched standard guidelines, we assessed the percentage of responses that fell within available guideline ranges in the UK using Pearson's chi-square tests. 


\section{INTERPRETATIONS OF NUTRIENT QUANTITIES}

To assess predictability of variations in interpretations, we used separate ANCOVAs to analyse the interpretations of each verbal quantity. In the ANCOVA, nutrient valence was used as a factor and BMI, attitudes towards healthy eating, frequency of label-checking, age, gender, level of education and ethnicity were used as covariates (in Experiment 2, we also added occupation and whether participant was a native English speaker). We also examined if attitudes, BMI, and label familiarity would interact with nutrient valence.

\section{$4 \quad$ Results}

\subsection{Participants' Interpretations of Verbal Quantities}

Participants' numerical estimates of verbal labels varied to a large extent (see Table 2). The difference between the numerical values provided for the different verbal quantities was significant in both experiments: $F(4,292)=322.89, p<.001, \eta^{2}=.82$ $($ Experiment 1$) ; F(4,3196)=1283.66, \mathrm{p}<.001, \eta^{2}{ }_{\mathrm{P}}=.62($ Experiment 2$)$. Backtranslations of numerical quantities into verbal ones generally matched the mean numerical estimates given for the translation of verbal into numerical quantities. The numerical values were consistently ranked in ascending order when assigned back to verbal quantities, Kendall's $w=.62, \chi^{2}(7, N=75)=325.25, p<.001$. Visual analogue scale perceptions of both verbal and numerical quantities varied greatly (see Figure 3).

\subsection{Alignment of Interpretations with Standard Guidelines}

Participants' numerical estimates of verbal quantities were much higher than the typical ranges in recommended guidelines, with $42-88 \%$ of interpretations exceeding recommended low and medium ranges (see Figure 4). While most estimates for high values tended to fall in the correct range by default, it should be noted that for positive nutrients, the $30 \%$ cut-off is a minimum value below which the declaration of 'high' 
INTERPRETATIONS OF NUTRIENT QUANTITIES

cannot be legally used. Therefore about $87 \%$ of participants in Experiment 1 and $75 \%$ in Experiment 2 overestimated these quantities of positive nutrients.

\subsection{Predictors of Variability in Interpretations}

We found no significant effect of any of the individual difference variables on the magnitude of numerical estimates for verbal quantities in Experiment 1 or 2 (Experiment 1: all $p \mathrm{~s}>.05$; Experiment 2: all $p \mathrm{~s}>.18$ ). Experiment 1 found no difference between interpretations for positive and negative nutrients, $F(1,62)<2.83, p$ $>.10, \eta_{\mathrm{p}}^{2}<.05$ for the five verbal quantities. However, in Experiment 2, numerical estimates for minerals were significantly higher than those for fat, $F(1,799)=104.54, p$ $<.001, \eta_{\mathrm{p}}^{2}=.01$. Full results of the ANCOVAs are provided in the Appendix (Tables A1 and A2).

\section{Discussion}

\subsection{Overestimation of verbal nutrient quantities}

Findings from two experiments showed that the interpretation of verbal labels varied greatly between individuals and that on average these interpretations did not match their intended meaning. For example, a low $\%$ indicated $5 \%$ of the GDA for one participant, but $40 \%$ for another. This is the first study to demonstrate this phenomenon in the context of nutrition labelling. Similar to findings for medical side effects (Berry, 2006; Webster et al., 2017b), but in contrast to those regarding climate change beliefs (Budescu et al., 2014), participants tended to overestimate the numerical value of all the verbal quantities. In the student sample, less than a quarter of interpretations were within the recommended range. In the nationwide sample, around half the interpretations exceeded guidelines. This could be because people naturally anchor verbal quantities to the full range of a percentage scale instead of the ranges provided by food labelling guidelines (which are skewed low, from 5-30\%). However, beyond the 


\section{INTERPRETATIONS OF NUTRIENT QUANTITIES}

proportion of people overestimating, the extent of overestimation was substantial in scale. For example, a high \% was commonly translated as around $68 \%$ in Experiment 1 and $48 \%$ in Experiment 2, which are both well over official cut-offs of $25-30 \%$ for high.

\subsection{Nutrient valence, but not personal characteristics, affected interpretations}

Experiment 2 also showed that people perceive verbal quantities of minerals to be larger than quantities of fat, although this did not occur when participants saw many nutrients one after another (Experiment 1). In line with a motivated reasoning account of quantity interpretation (Kunda, 1990), participants might wish to believe that they were not consuming too much of a negative nutrient, but were eating more of a positive one. However, we did not find evidence that individual differences in motivation to eat healthily affected participants' interpretations, nor did any other individual difference variable predict participants' estimations. This could reflect that individual predictors for making healthier choices (e.g., motivation to eat healthily; Hearty, McCarthy, Kearney, \& Gibney, 2007) or better health literacy (e.g., education level: Sinclair, Hammond, \& Goodman, 2013; familiarity with labels: Gigerenzer, Hertwig, van den Broek, Fasolo, \& Katsikopoulos, 2005) do not guard against overestimation of verbal quantities. Our findings contrast with studies in verbal probabilities, where differences in attitudes significantly predicted interpretational variability (Budescu et al., 2014), and studies in verbal frequencies, where gender, ethnicity, and education predicted overestimations of risk frequencies (Webster et al., 2017b). This could be because attitudes are less predictive of quantity perception for food than for contexts such as climate change, where one's attitude is more closely related to their beliefs (Hornsey, Harris, Bain, \& Fielding, 2016). More frequent label use may also not offer the right feedback to inform people that they are overestimating verbal quantities. Therefore, the individual difference variables we measured may not have fully captured the factors that 


\section{INTERPRETATIONS OF NUTRIENT QUANTITIES}

might explain variance in interpretations. Beliefs about specific nutrients and their contributions to health (e.g., Oakes, 2005b) might explain tendencies to overestimate quantities more than general attitudes towards eating healthily. It would be good to investigate further if people's cognitions about different categories of nutrients can form a stable factor to determine their tendency to overestimate a quantity.

\subsection{Limitations}

Two aspects of the studies might limit generalisation of the results. First, the use of survey methodology is subject to selection bias. Although we tackled a larger sample with a wider range of educational levels in Experiment 2, which addressed a limitation of the first experiment (with a primarily undergraduate-based sample), a survey panel inevitably selects members of the population with access and motivation to complete online surveys. Such samples may potentially have higher numeracy and health literacy (Couper \& Singer, 2009; Duffy, Smith, Terhanian, \& Bremer, 2005). This limits the representativeness of our sample to less numerate and literate segments of the population. We could expect that in such groups, lower numeracy and health literacy might predict more substantial overestimations than we found in our sample. On the other hand, participants in online surveys may also include a larger proportion of online shoppers, who are less frequent seekers of nutritional information (Benn, Webb, Chang, \& Reidy, 2015). Additional research is needed to validate our findings among less technologically-adept participants.

A second limitation of the studies was that presenting the label interpretation task in isolation might also remove some of the contextual knowledge that consumers typically use to judge food - for example, brand information (Cavanagh \& Forestell, 2013), health claims (Williams, 2005), and other visual cues (e.g., colour-coded labels; Jones \& Richardson, 2007). In a real shopping or food consumption context, such 


\section{INTERPRETATIONS OF NUTRIENT QUANTITIES}

information might help to reduce variation in interpretations. Future research might thus follow up on whether people's overestimations of verbal quantities extend to interpretations made at point of sale.

\section{Implications for Research and Practice}

Given the large variation among individuals in interpreting verbal quantities, the advisability of using verbal labels seems questionable. However, verbal expressions remain widely used because they are more natural in communication (Zimmer, 1983) and do not require precise estimation of quantities. For example, GDA information is meant as a recommended guideline based on population averages for daily required nutrient intake (UK Food and Drink Federation, 2009) and so it would be inaccurate to assume a '5\%' GDA label indicates exactly 5\% of one's GDA. Here, a 'low \%', thanks to its vagueness, may actually convey the information more accurately, provided its range is interpreted as intended.

The tendency to overestimate verbal nutrient quantities presents implications for people's understanding about their diet quality. Although the scales for nutrition labelling reflect real-world distributions (e.g., a food would typically not contribute more than $30 \%$ of one's GDA of a nutrient; Rayner, Scarborough, \& Williams, 2004), if people do not realise that the guideline ranges are scaled towards small quantities, they

will still misinterpret the verbal terms. They could thus believe they are consuming more of a particular nutrient than they actually are. This would be primarily a problem for under-consumption of food groups like vitamins, minerals, and fibre. The belief that one's own diet already meets dietary recommendations for these food groups can prevent one from taking action to improve dietary behaviour (Lechner, Brug, De Vries, van Assema, \& Mudde, 1998). For example, manufacturers can declare a food high in fibre if it reaches $30 \%$ of the GDA. However, if one believes it provides $70 \%$ of a day's 


\section{INTERPRETATIONS OF NUTRIENT QUANTITIES}

recommended fibre consumption, one would mistakenly assume that one has almost reached their fibre target for the day, and not seek to improve their fibre intake. Fibre consumption in the general population is far below recommended amounts (Guiné et al., 2016); $97 \%$ of adults in our sample population already do not meet daily fibre targets (NatCen Social Research, 2015). Both overconsumption of negative nutrients and under-consumption of positive ones impact health (UK Department of Health, 2015), so it is important for future work to determine whether, and to what extent overestimating the nutrient content of one's food affects subsequent consumption decisions.

A further problem about misinterpreting verbal quantities arises because information about different nutrients is often presented with different formats on the same product. When comparing between verbal and numerical quantities, people may reach incorrect conclusions about the levels of nutrients within one product. For example, a breakfast cereal that is 'high in fibre' while providing a 30\% GDA of sugar might seem to have a lot more fibre than sugar when in fact both nutrients contribute equally to their respective GDAs. Differences in interpretation of verbal and numerical labels can also result in erroneous comparisons between two products that use a verbal and numerical quantity respectively. For example, one might mistakenly choose cereal with a $12 \%$ GDA of fat as healthier than a low-fat cereal if one believes 'low' to indicate $20 \%$. Although past work indicates that consumers can use labels in the same format to pick between products with higher or lower nutrient contents (Hersey et al., 2013), it is not known how they would perform when comparing between foods with different label formats.

A potential solution might be to standardise GDA labelling across all food products to include both verbal and numerical information (e.g., the hybrid Traffic Light system; Limb, 2012). Dual (verbal-numerical) scales are intended to increase 


\section{INTERPRETATIONS OF NUTRIENT QUANTITIES}

interpretational consistency (Budescu et al., 2012), however there is no guarantee that people will not rely on only one format or the other, especially if it is unclear how the scale values are derived. Greater reliance on text over numerical information is also seen in people with low numeracy (Dieckmann, Slovic, \& Peters, 2009), who tend to prefer non-numeric information (Peters, 2012; Reyna, Nelson, Han, \& Dieckmann, 2009). Nevertheless, dual-scale labels may pose an advantage over number-only labels, which are more likely to be ignored due to the higher cognitive effort involved in processing them (Cowburn \& Stockley, 2005).

Alternatively, research from the medical domain advocate the use of graphic representations of quantitative information, for instance, using discrete frequencies to represent probabilities (e.g., icon arrays; Zipkin et al., 2014). Such formats facilitate understanding of health risks, particularly among less numerate or literate segments of the population (Gigerenzer \& Kolpatzik, 2017). In practice, consumers often make food choice decisions quickly (Celnik, Gillespie, \& Lean, 2012), and food packaging has limited space available to provide information. Alternative formats attempt to address these issues by interpreting the food product for consumers (Maubach, Hoek, \& Mather, 2014). For example, a label may classify products as either healthy or not (e.g., tick logos: Scott \& Worsley, 1994; the Smart Choices label: Roberto et al., 2012), give a product a healthiness score (e.g., the Star rating; Maubach et al., 2014), or use colour coding to draw attention to the level of a nutrient (e.g., Traffic Light systems; Mejean, Macouillard, Peneau, Hercberg, \& Castetbon, 2013).

Interpretive labels could help people understand labels by tapping into their tendency to form categorical representations of quantitative information-for instance, they might form a rough summary of the information, such as 'the food has fibre' or 'the food has fat' to make their decision (Blalock et al., 2016). However, food 


\section{INTERPRETATIONS OF NUTRIENT QUANTITIES}

information must still align with consumer expectations if they are to provide an accurate picture of the quality of one's diet (Celnik et al., 2012). For example, the colour bands in the Traffic Light system (red, amber, and green) correspond to verbal bands of high, medium, and low (UK Department of Health, 2016). Even if colourcoded labels are easier to process (Siegrist, Leins-Hess, \& Keller, 2015), consumer misinterpretations of the low, medium, and high bands could still lead to mistaken assumptions about the quality of their diets. Thus, the issue of whether verbal bands (or other interpretive text) match psychologically equivalent numerical values remains important. Current guideline ranges for determining verbal banding were developed based on scientific research on nutritional values suitable for populations, and allow for discrimination between the low-skewed GDA percentages typically seen on individual foods (Rayner et al., 2004). However, guidelines for verbal labels are determined by GDA percentages for some, but not all nutrients (UK Department of Health, 2011, 2016). This increases the likelihood that people will revert to natural interpretations when reading nutrition labels. These interpretations appear to be independent of the specifications of food science, show little discrimination between small numerical differences, and are difficult to override (Budescu et al., 2012; Webster, Weinman, \& Rubin, 2017b). Indeed, existing public education about verbal banding on nutrition labels (UK Food Standards Agency, 2007, 2008) did not enable our participants to suppress their natural interpretations of verbal and numerical quantities.

Recalibrating the ranges assigned to verbal quantities could balance the ability to easily compare food products with the goal of boosting nutritional understanding (Hertwig \& Grüne-Yanoff, 2017) by harnessing the intuitive understanding of what these quantities mean. However, given the great inter-individual variability in interpretations, identifying the best interpretive range for verbal nutrient quantities is 


\section{INTERPRETATIONS OF NUTRIENT QUANTITIES}

challenging in practice. Nonetheless, our study suggests two immediate concerns with current guidelines that could be addressed. First, guidelines on the use of verbal quantities such as 'high' for positive nutrients could be pegged higher to avoid problematic overestimation of positive nutrient consumption. Second, interpretational guidelines could be standardised across nutrients and products to facilitate comparisons. We also hope that our work can stimulate more empirical research to support the continued refinement of nutritional guidelines. 
INTERPRETATIONS OF NUTRIENT QUANTITIES

\section{References}

Benn, Y., Webb, T. L., Chang, B. P. I., \& Reidy, J. (2015). What information do consumers consider, and how do they look for it, when shopping for groceries online? Appetite, 89, 265-273. doi: 10.1016/j.appet.2015.01.025

Berry, D. C. (2006). Verbal labels can triple perceived risk in clinical trials. Therapeutic Innovation and Regulatory Science, 40, 249-258. doi:

\section{$10.1177 / 009286150604000302$}

Berry, D. C., Raynor, D. K., \& Knapp, P. R. (2003). Communicating risk of medication side effects: an empirical evaluation of EU recommended terminology. Psychology, Health \& Medicine, 8, 251-263. doi: $10.1080 / 1354850031000135704$

Blalock, S. J., Sage, A., Bitonti, M., Patel, P., Dickinson, R., \& Knapp, P. (2016). Communicating information concerning potential medication harms and benefits: What gist do numbers convey? Patient Education and Counseling, 99, 1964-1970. doi: 10.1016/j.pec.2016.07.022

Blitstein, J. L., \& Evans, W. D. (2006). Use of nutrition facts panels among adults who make household food purchasing decisions. Journal of Nutrition Education \& Behavior, 38, 360-364. doi: 10.1016/j.jneb.2006.02.009

Budescu, D. V., Por, H.-H., \& Broomell, S. B. (2012). Effective communication of uncertainty in the IPCC reports. Climatic Change, 113, 181-200. doi: $10.1007 / \mathrm{s} 10584-011-0330-3$

Budescu, D. V., Por, H.-H., Broomell, S. B., \& Smithson, M. (2014). The interpretation of IPCC probabilistic statements around the world. Nature Climate Change, 4, 508-512. doi: 10.1038/NCLIMATE2194 


\section{INTERPRETATIONS OF NUTRIENT QUANTITIES}

Cavanagh, K. V., \& Forestell, C. A. (2013). The effect of brand names on flavor perception and consumption in restrained and unrestrained eaters. Food Quality and Preference, 28, 505-509. doi: 10.1016/j.foodqual.2012.12.004

Celnik, D., Gillespie, L., \& Lean, M. E. J. (2012). Time-scarcity, ready-meals, ill-health and the obesity epidemic. Trends in Food Science and Technology, 27, 4-11. doi: 10.1016/j.tifs.2012.06.001

Collins, P. J., \& Hahn, U. (2018). Communicating and reasoning with verbal probability expressions. Psychology of Learning and Motivation, 69, 67-105.

Couper, M. P., \& Singer, E. (2009). The role of numeracy in informed consent for surveys. Journal of Empirical Research on Human Research Ethics, 4, 17-26. doi: 10.1525/jer.2009.4.4.17

Cowburn, G., \& Stockley, L. (2005). Consumer understanding and use of nutrition labelling: A systematic review. Public Health Nutrition, 8, 21-28. doi: 10.1079/PHN2004666

Craig, R., \& Shelton, N. (2008). Health survey for England 2007: Healthy lifestyles: knowledge, attitudes and behaviour. Summary of key findings. Leeds, UK: Public Health England.

Crockett, R. A., King, S. E., Marteau, T. M., Prevost, A. T., Bignardi, G., Roberts, N. W., et al. (2018). Nutritional labelling for healthier food or non-alcoholic drink purchasing and consumption. Cochrane Database Systematic Reviews, 27, CD009315. doi: 10.1002/14651858.CD009315.pub2.

Dieckmann, N. F., Slovic, P., \& Peters, E. M. (2009). The use of narrative evidence and explicit likelihood by decisionmakers varying in numeracy. Risk Analysis, 29, 1473-1488. doi: 10.1111/j.1539-6924.2009.01279.x 


\section{INTERPRETATIONS OF NUTRIENT QUANTITIES}

Du, N., \& Stevens, K. (2011). Numeric-to-verbal translation of probability expressions in SFAS 5. Managerial Auditing Journal, 26, 248-262. doi: $10.1108 / 02686901111113190$

Duffy, B., Smith, K., Terhanian, G., \& Bremer, J. (2005). Comparing data from online and face-toface surveys. International Journal of Market Research, 47, 615-639. doi: $10.1177 / 147078530504700602$

Gardner, P. H., McMillan, B., Raynor, D. K., Woolf, E., \& Knapp, P. (2011). The effect of numeracy on the comprehension of information about medicines in users of a patient information website. Patient Education and Counseling, 83, 398-403. doi: 10.1016/j.pec.2011.05.006

Gigerenzer, G., Hertwig, R., van den Broek, E., Fasolo, B., \& Katsikopoulos, K. V. (2005). "A 30\% chance of rain tomorrow": How does the public understand probabilistic weather forecasts? Risk Analysis, 25, 623-629. doi: 10.1111/j.15396924.2005.00608.x

Gigerenzer, G., \& Kolpatzik, K. (2017). How new fact boxes are explaining medical risk to millions. British Medical Journal, 357, j2460. doi: 10.1136/bmj.j2460

Grunert, K. G., Fernández-Celemín, L., Wills, J. M., Storcksdieck genannt Bonsmann, S., \& Nureeva, L. (2010). Use and understanding of nutrition information on food labels in six European countries. Zeitschrift Fur Gesundheitswissenschaften, 18, 261-277. doi: 10.1007/s10389-009-0307-0

Grunert, K. G., Wills, J. M., \& Fernandez-Celemin, L. (2010). Nutrition knowledge, and use and understanding of nutrition information on food labels among consumers in the UK. Appetite, 55, 177-189. doi: 10.1016/j.appet.2010.05.045

Guiné, R. P. F., Duarte, J., Ferreira, M., Correia, P., Leal, M., Rumbak, I., et al. (2016). Attitudes towards dietary fibre on a multicultural basis: A fibre study 


\section{INTERPRETATIONS OF NUTRIENT QUANTITIES}

framework. Current Nutrition and Food Science, 12, 132-141. doi:

$$
10.2174 / 157340131202160412171430
$$

Harris, A. J. L., Corner, A., \& Hahn, U. (2009). Estimating the probability of negative events. Cognition, 110, 51-64. doi: 10.1016/j.cognition.2008.10.006

Hawley, K. L., Roberto, C. A., Bragg, M. A., Liu, P. J., Schwartz, M. B., \& Brownell, K. D. (2013). The science on front-of-package food labels. Public Health Nutrition, 16, 430-439. doi: 10.1017/S1368980012000754

Hearty, Á. P., McCarthy, S. N., Kearney, J. M., \& Gibney, M. J. (2007). Relationship between attitudes towards healthy eating and dietary behaviour, lifestyle and demographic factors in a representative sample of Irish adults. Appetite, 48, 111. doi: 10.1016/j.appet.2006.03.329

Hersey, J. C., Wohlgenant, K. C., Arsenault, J. E., Kosa, K. M., \& Muth, M. K. (2013). Effects of front-of-package and shelf nutrition labeling systems on consumers. Nutrition Reviews, 71, 1-14. doi: 10.1111/nure.12000

Hertwig, R., \& Grüne-Yanoff, T. (2017). Nudging and boosting: Steering or empowering good decisions. Perspectives on Psychological Science, 1-14. doi: $10.1177 / 1745691617702496$

Hibbard, J. H., Peters, E., Dixon, A., \& Tusler, M. (2007). Consumer competencies and the use of comparative quality information. It isn't just about literacy. Medical Care Research \& Review, 64. doi: 10.1177/1077558707301630

Hornsey, M. J., Harris, E. A., Bain, P. G., \& Fielding, K. S. (2016). Meta-analyses of the determinants and outcomes of belief in climate change. Nature Climate Change, 6, 622-626.

Jensen Hjermstad, M., Fayers, P. M., Haugen, D. F., Caraceni, A., Hanks, G. W., Loge, J. H., et al. (2011). Studies comparing numerical rating scales, verbal rating 


\section{INTERPRETATIONS OF NUTRIENT QUANTITIES}

scales, and visual analogue scales for assessment of pain intensity in adults: A systematic literature review. Journal of Pain and Symptom Management, 41, 1073-1093. doi: 10.1016/j.jpainsymman.2010.08.016

Jones, G., \& Richardson, M. (2007). An objective examination of consumer perception of nutrition information based on healthiness ratings and eye movements. Public Health Nutr, 10, 238-244. doi: 10.1017/S1368980007258513

Juanchich, M., Sirota, M., \& Butler, C. L. (2012). The perceived functions of linguistic risk quantifiers and their effect on risk, negativity perception and decision making. Organizational Behavior and Human Decision Processes, 118, 72-81. doi: 10.1016/j.obhdp.2012.01.002

Knapp, P. R., Gardner, P. H., \& Woolf, E. (2015). Combined verbal and numerical expressions increase perceived risk of medicine side-effects: A randomized controlled trial of EMA recommendations. Health Expectations, 19, 264-274. doi: $10.1111 /$ hex.12344

Knapp, P., Raynor, D. K., \& Berry, D. C. (2004). Comparison of two methods of presenting risk information to patients about the side effects of medicines. $B M J$ Quality and Safety, 13, 176-180. doi: 10.1136/qshc.2003.009076

Kunda, Z. (1990). The case for motivated reasoning. Psychological Bulletin, 108, 480498. doi: 10.1037/0033-2909.108.3.480

Lechner, L., Brug, J., De Vries, H., van Assema, P., \& Mudde, A. (1998). Stages of change for fruit, vegetable and fat intake: consequences of misconception. Health Education Research, 13, 1-11. doi: 10.1093/her/13.1.1-a

Limb, M. (2012). Food labelling scheme agreed with retailers to remain voluntary. British Medical Journal, 345, e7212. doi: 10.1136/bmj.e7212 


\section{INTERPRETATIONS OF NUTRIENT QUANTITIES}

Lipkus, I. M., \& Peters, E. (2009). Understanding the role of numeracy in health: Proposed theoretical framework and practical insights. Health Education \& Behavior, 36, 1065-1081. doi: 10.1177/1090198109341533

Malam, S., Clegg, S., Kirwan, S., \& McGinigal, S. (2009). Comprehension and use of UK nutrition signpost labelling schemes. London, UK: BMRB.

Maubach, N., Hoek, J., \& Mather, D. (2014). Interpretive front-of-pack nutrition labels. Comparing competing recommendations. Appetite, 82, 67-77. doi:

10.1016/j.appet.2014.07.006

Mejean, C., Macouillard, P., Peneau, S., Hercberg, S., \& Castetbon, K. (2013). Consumer acceptability and understanding of front-of-pack nutrition labels. $J$ Hum Nutr Diet, 26, 494-503. doi: 10.1111/jhn.12039

NatCen Social Research. (2015). National diet and nutrition survey years. London, UK: UK Data Service.

Oakes, M. E. (2005a). Bad company: The addition of sugar, fat, or salt reduces the perceived vitamin and mineral content of foods. Food Quality \& Preference, 16, 111-119. doi: 10.1016/j.foodqual.2004.02.007

Oakes, M. E. (2005b). Stereotypical thinking about foods and perceived capacity to promote weight gain. Appetite, 44, 317-324. doi: 10.1016/j.appet.2005.03.010

Parmenter, K., Waller, J., \& Wardle, J. (2000). Demographic variation in nutrition knowledge in England. Health Education Research, 15, 163-174. doi: $10.1093 /$ her/15.2.163

Peters, E. (2012). Beyond comprehension: The role of numeracy in judgments and decisions. Current Directions in Psychological Science, 21, 31-35. doi: $10.1177 / 0963721411429960$ 


\section{INTERPRETATIONS OF NUTRIENT QUANTITIES}

Piercey, M. D. (2009). Motivated reasoning and verbal vs. numerical probability assessment: Evidence from an accounting context. Organizational Behavior \& Human Decision Processes, 108, 330-341. doi: 10.1016/j.obhdp.2008.05.004

Rayner, M., Boaz, A., \& Higginson, C. (2001). Consumer use of health-related endorsements on food labels in the United Kingdom and Australia. Journal of Nutrition Education, 33, 24-30. doi: 10.1016/S1499-4046(06)60006-7

Rayner, M., Scarborough, P., \& Williams, C. (2004). The origin of Guideline Daily Amounts and the Food Standards Agency's guidance on what counts as 'a lot' and 'a little'. Public Health Nutrition, 7, 549-556. doi: 10.1079/PHN2003552

Reyna, V. F., Nelson, W. L., Han, P. K., \& Dieckmann, N. F. (2009). How numeracy influences risk comprehension and medical decision making. Psychological Bulletin, 135, 943-973. doi: 10.1037/a0017327

Roberto, C. A., Shivaram, M., Martinez, O., Boles, C., Harris, J. L., \& Brownell, K. D. (2012). The Smart Choices front-of-package nutrition label. Influence on perceptions and intake of cereal. Appetite, 58, 651-657. doi: 10.1016/j.appet.2012.01.003

Scott, V., \& Worsley, A. F. (1994). Ticks, claims, tables and food groups: a comparison for nutrition labelling. Health Promotion International, 9, 27-37. doi: 10.1093/heapro/9.1.27

Shannon, B. (1994). Nutrition labelling: Putting the consumer first. British Food Journal, 96, 40-44. doi: 10.1108/00070709410061087

Siegrist, M., Leins-Hess, R., \& Keller, C. (2015). Which front-of-pack nutrition label is the most efficient one? The results of an eye-tracker study. Food Quality \& Preference, 39, 183-190. doi: 10.1016/j.foodqual.2014.07.010 


\section{INTERPRETATIONS OF NUTRIENT QUANTITIES}

Sinclair, S., Hammond, D., \& Goodman, S. (2013). Sociodemographic differences in the comprehension of nutritional labels on food products. J Nutr Educ Behav, 45, 767-772. doi: 10.1016/j.jneb.2013.04.262

Sirota, M., \& Juanchich, M. (2015). A direct and comprehensive test of two postulates of politeness theory applied to uncertainty communication. Judgment and Decision Making, 10, 232-240.

Steptoe, A., Pollard, T. M., \& Wardle, J. (1995). Development of a measure of the motives underlying the selection of food: The food choice questionnaire. Appetite, 25, 267-284. doi: 10.1006/appe.1995.0061

Storcksdieck genannt Bonsmann, S., Férnández Celemín, L., Larrañaga, A., Egger, S., Wills, J. M., Hodgkins, C., et al. (2010). Penetration of nutrition information on food labels across the EU-27 plus Turkey. European Journal of Clinical Nutrition, 64, 1379-1385. doi: 10.1038/ejen.2010.179

Temple, N. J., \& Fraser, J. (2014). Food labels: A critical assessment. Nutrition, 30, 257-260. doi: 10.1016/j.nut.2013.06.012

Theil, M. (2002). The role of translations of verbal into numerical probability expressions in risk management: a meta-analysis. Journal of Risk Research, 5, 177-186. doi: 10.1080/13669870110038179

UK Department of Health. (2011). Nutrition and health claims. Guidance to compliance with Regulation (EC) 1924/2006 on nutrition and health claims made on foods.

UK Department of Health (2015). Food and diet. Retrieved 19 October, 2017, from https://www.nhs.uk/LiveWell/Goodfood/Pages/goodfoodhome.aspx

UK Department of Health. (2016). Technical guidance on nutrition labelling.

UK Food and Drink Federation. (2009). Science behind guideline daily amounts. 


\section{INTERPRETATIONS OF NUTRIENT QUANTITIES}

UK Food Standards Agency, F. (2007). Food. Using traffic lights to make healthier choices.

UK Food Standards Agency. (2008). Food labels. More informed choices.

Webster, R. K., Weinman, J., \& Rubin, G. J. (2017a). How does the side-effect information in patient information leaflets influence peoples' side-effect expectations? A cross-sectional national survey of 18- to 65-year-olds in England. Health Expectations, 20, 1411-1420. doi: 10.1111/hex.12584

Webster, R. K., Weinman, J., \& Rubin, G. J. (2017b). People's understanding of verbal risk descriptors in patient information leaflets: A cross-sectional national survey of 18- to 65-year-olds in England. Drug Safety, 1-12. doi: 10.1007/s40264-017$0542-1$

Williams, P. G. (2005). Consumer understanding and use of health claims for foods. Nutrition Reviews, 63, 256-264.

Zimmer, A. C. (1983). Verbal vs. numerical processing of subjective probabilities. In R. W. Scholz (Ed.), Advances in Psychology (Vol. 16, pp. 159-182). London, UK: Elsevier.

Zipkin, D. A., Umscheid, C. A., Keating, N. L., Allen, E., Aung, K., Beyth, R., et al. (2014). Evidence-based risk communication: A systematic review. Annals of Internal Medicine, 161, 270-280. doi: 10.7326/M14-0295 


\section{INTERPRETATIONS OF NUTRIENT QUANTITIES}

Table 1.

Socio-demographic characteristics of the sample in Experiment 2

\begin{tabular}{|c|c|c|}
\hline & $\bar{N}$ & Percentage of Sample \\
\hline \multicolumn{3}{|l|}{ Age Range } \\
\hline $18-24$ & 54 & 6.8 \\
\hline $25-34$ & 138 & 17.4 \\
\hline $35-44$ & 147 & 18.5 \\
\hline $45-54$ & 151 & 19.0 \\
\hline $55-64$ & 148 & 18.6 \\
\hline $65-74$ & 157 & 19.7 \\
\hline \multicolumn{3}{|l|}{ Ethnicity } \\
\hline White & 734 & 92.2 \\
\hline Asian & 34 & 4.3 \\
\hline Black & 14 & 1.8 \\
\hline Mixed & 10 & 1.3 \\
\hline Other & 4 & 0.5 \\
\hline \multicolumn{3}{|l|}{ Employment Status } \\
\hline Full-time & 330 & 41.3 \\
\hline Part-time & 118 & 14.8 \\
\hline Self-employed & 42 & 5.3 \\
\hline Student & 22 & 2.8 \\
\hline Unemployed & 98 & 12.3 \\
\hline Retired & 190 & 23.8 \\
\hline \multicolumn{3}{|l|}{ Highest Education Level } \\
\hline High school or equivalent & 339 & 42.4 \\
\hline Degree or higher & 285 & 35.7 \\
\hline Apprenticeship & 35 & 4.4 \\
\hline Other Qualifications & 96 & 12.0 \\
\hline No Qualifications & 43 & 5.4 \\
\hline \multicolumn{3}{|l|}{ Frequent Nutrition Label Use } \\
\hline Strongly disagree & 73 & 9.2 \\
\hline Disagree & 81 & 10.2 \\
\hline Somewhat disagree & 98 & 12.3 \\
\hline Neither agree nor disagree & 151 & 19.0 \\
\hline Somewhat agree & 220 & 27.7 \\
\hline Agree & 119 & 15.0 \\
\hline \multirow[t]{2}{*}{ Strongly agree } & 53 & 6.7 \\
\hline & $M$ & $S D$ \\
\hline Attitudes towards Healthy Eating & 4.7 & 1.1 \\
\hline Body Mass Index (BMI) & 27.4 & 6.7 \\
\hline
\end{tabular}

Note. Frequency of nutrition label use was measured via agreement on a 7-point Likert scale to the statement, 'I often use nutritional labels to determine the healthiness of 


\section{INTERPRETATIONS OF NUTRIENT QUANTITIES}

food.' Attitudes towards healthy eating were measured using four questions from the healthy eating motivation section of the Food Choice Questionnaire (Steptoe, Pollard, \& Wardle, 1995): 'It is important that the food I eat keeps me healthy', 'It is important that the food I eat helps me control my weight', 'I eat what I like and I do not worry about healthiness of food', and 'The healthiness of food has little impact on my food choices' (last two questions reverse-coded), with responses on a 7-point Likert scale (1: strongly disagree, 7: strongly agree). BMI was calculated from participants' selfreported height and weight. 
INTERPRETATIONS OF NUTRIENT QUANTITIES

Table 2.

Interpretations of Verbal and Numerical Quantities in Experiments 1 and 2

$$
\text { Experiment } 1(N=82)
$$

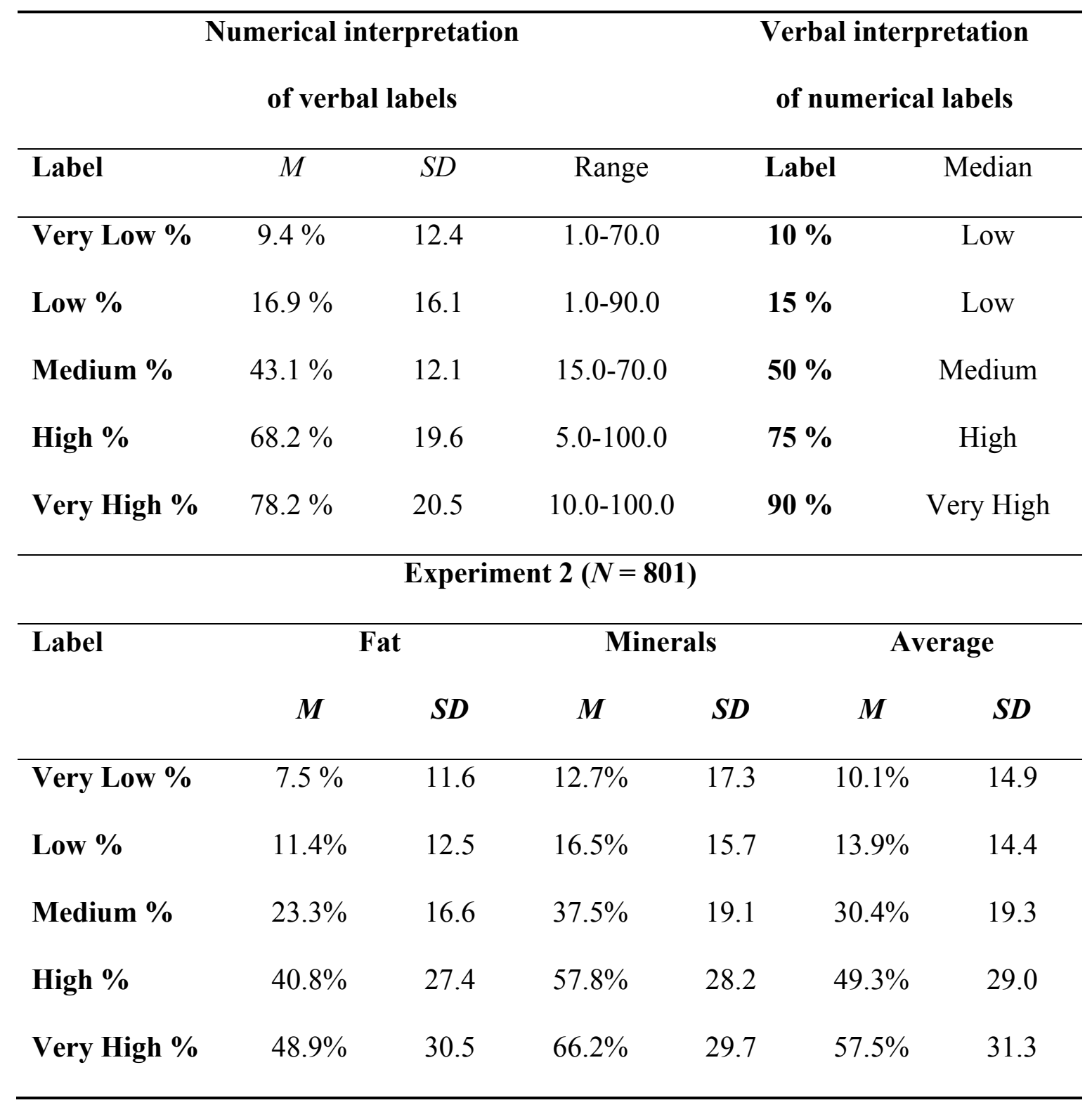




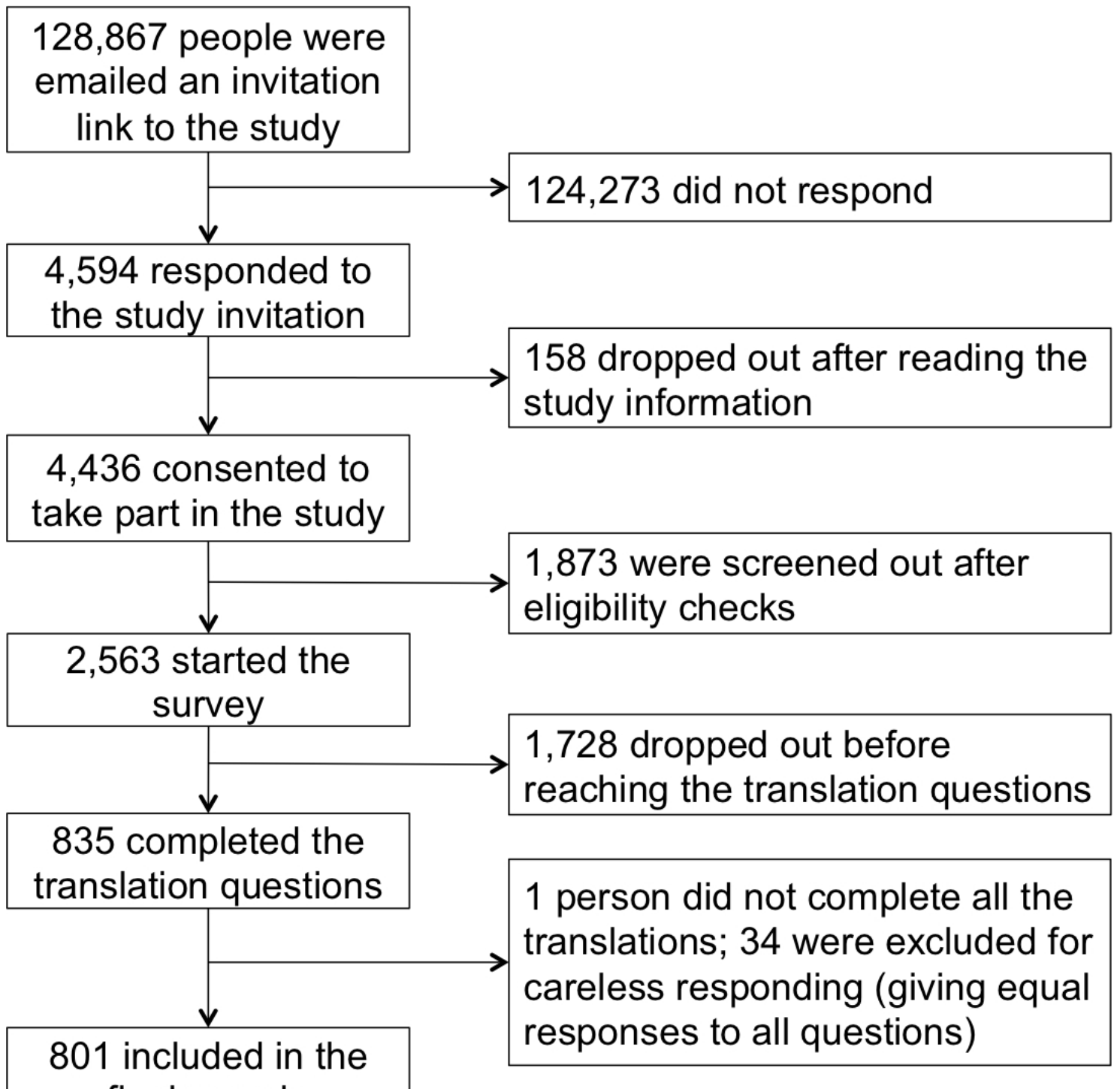

Figure 1. Flowchart of participant selection and exclusions in Experiment 2.

Note. Eligibility checks on participants' socio-demographics (age, gender, occupation, highest education level, and region of residence) were conducted to ensure population subgroups were proportionately represented. Participants who gave translated the five verbal quantifiers into equal numerical amounts were excluded. 


\section{INTERPRETATIONS OF NUTRIENT QUANTITIES}

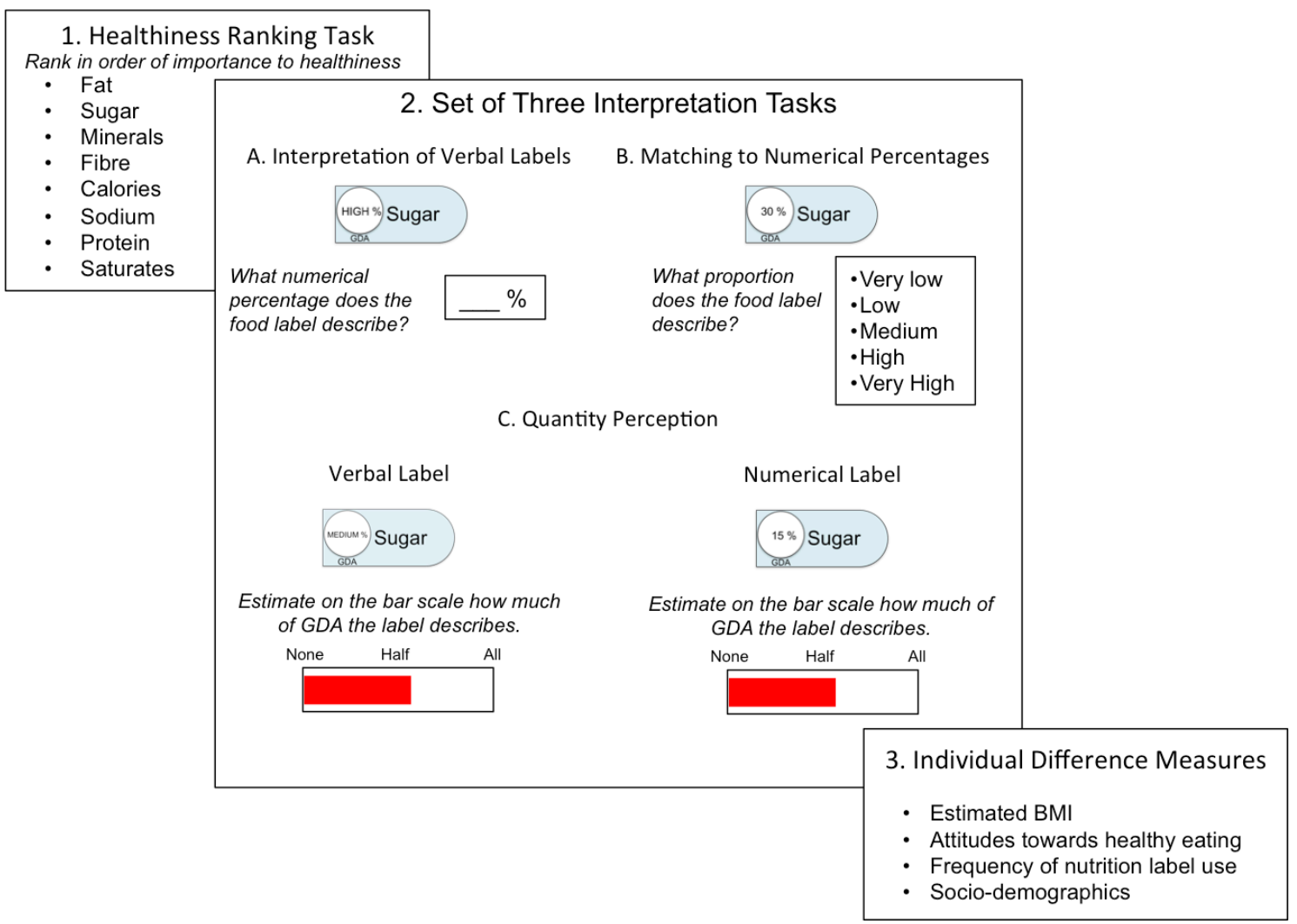

Figure 2. Structure of experimental tasks in Experiment 1.

Note. A sugar label is depicted as an example. In Experiment 1, each participant saw a different nutrient for each quantity. In Experiment 2, only the verbal label interpretation task was used, and participants saw either fat or minerals only.

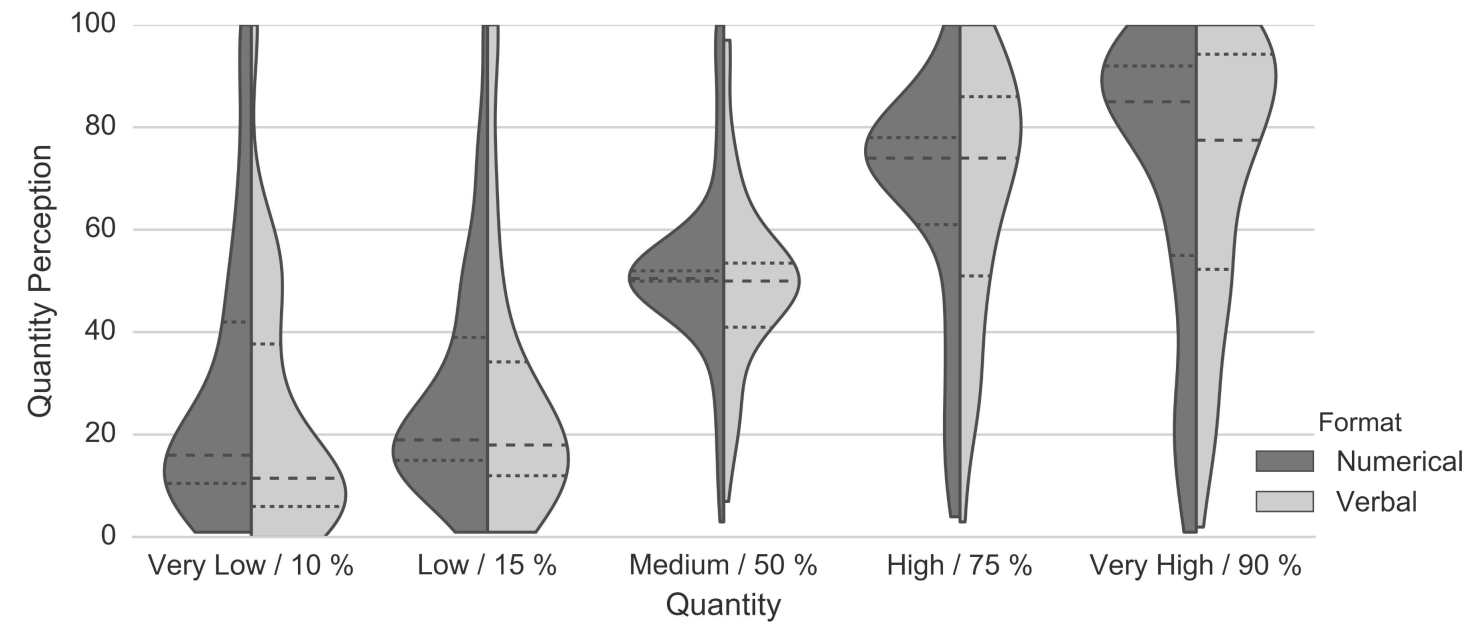

Figure 3. Distribution of estimates on the visual analogue scale for verbal and numerical GDA labels. Dotted lines indicate the medians and inter-quartile ranges. 


\section{INTERPRETATIONS OF NUTRIENT QUANTITIES}

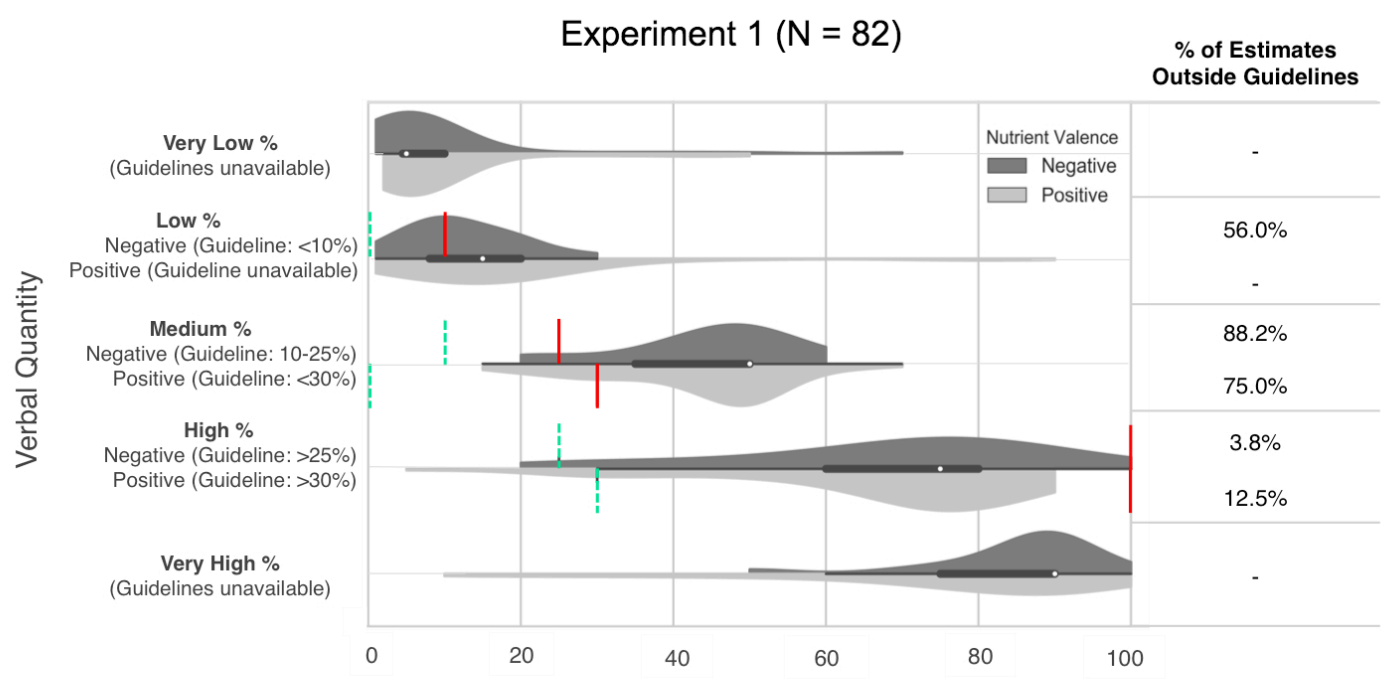

Experiment $2(\mathrm{~N}=801)$

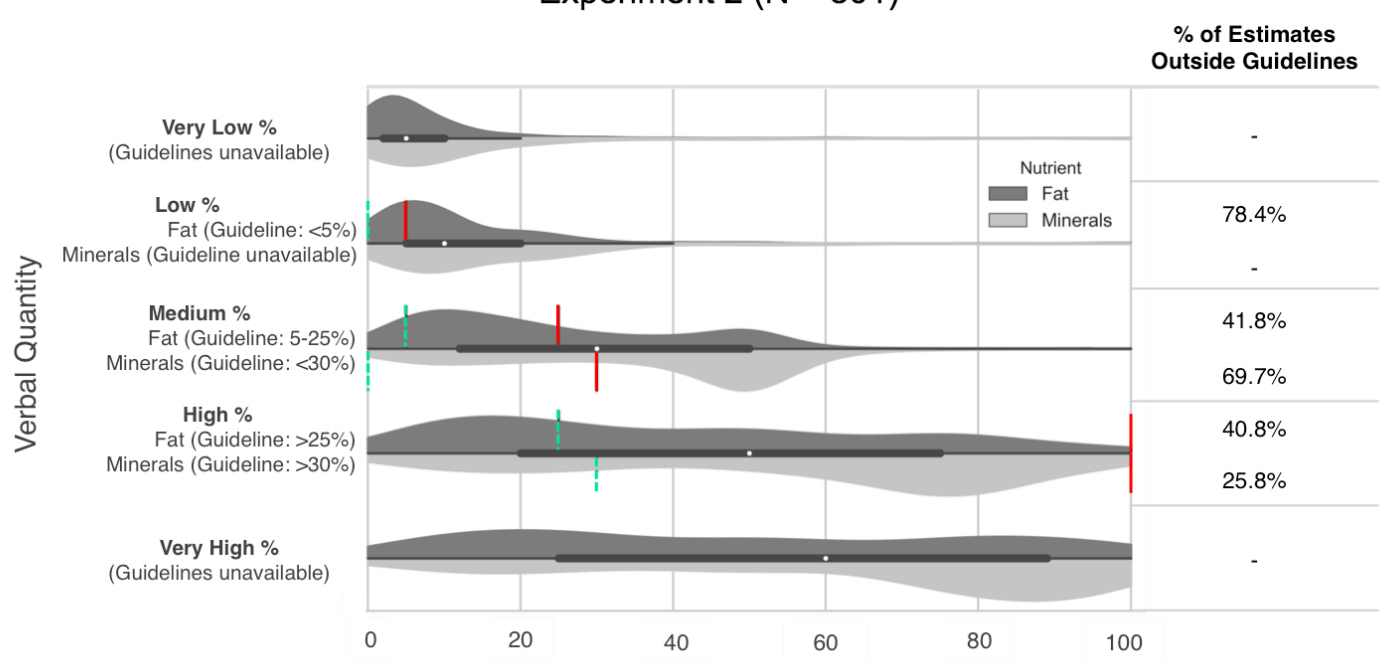

Figure 4. Distribution of verbal quantity interpretations in Experiments $1(N=82)$ and 2

$(N=801)$, and their fit with recommended guidelines (solid red lines indicate upper

limits and dotted green lines indicate lower limits).

Note. The guideline values for fat and minerals were estimated from UK Department of

Health $(2011,2016)$. For minerals, only the cut-off between medium and high $\%$ was available. 
INTERPRETATIONS OF NUTRIENT QUANTITIES

\section{Appendix}

Table A1

F-and p-values in the ANCOVAs for Interpretations of Verbal Labels in Experiment 1 $(d f=1,59)$

\begin{tabular}{|c|c|c|c|c|c|c|c|c|c|c|}
\hline \multirow[t]{3}{*}{ Factor } & \multicolumn{10}{|c|}{ Quantity } \\
\hline & \multicolumn{2}{|c|}{ Very Low } & \multicolumn{2}{|c|}{ Low } & \multicolumn{2}{|c|}{ Medium } & \multicolumn{2}{|c|}{ High } & \multicolumn{2}{|c|}{ Very High } \\
\hline & $\boldsymbol{F}$ & $p$ & $\boldsymbol{F}$ & $p$ & $\boldsymbol{F}$ & $p$ & $\boldsymbol{F}$ & $p$ & $\boldsymbol{F}$ & $p$ \\
\hline$\overline{\text { Nutrient valence }}$ & .11 & .739 & 1.23 & .271 & .01 & .916 & .63 & .432 & 1.04 & .312 \\
\hline Frequency of label use & .25 & .621 & .53 & .471 & $<.001$ & .999 & 2.46 & .122 & .03 & .855 \\
\hline Eating attitude & .64 & .426 & .24 & .629 & 1.60 & .211 & .28 & .601 & $<.001$ & .999 \\
\hline BMI & 3.59 & .063 & .59 & .445 & .52 & .474 & 2.55 & .116 & .44 & .509 \\
\hline Gender & 3.31 & .074 & 2.64 & .110 & .37 & .546 & .27 & .607 & .001 & .977 \\
\hline Age & .49 & .378 & 1.55 & .218 & .02 & .887 & .37 & .55 & .14 & .711 \\
\hline Education level & 2.93 & .092 & 2.57 & .114 & .34 & .561 & .36 & .548 & .10 & .754 \\
\hline Ethnicity & .25 & .621 & .06 & .806 & .68 & .414 & .25 & .617 & .24 & .628 \\
\hline Nutrient valence $x$ & .13 & .717 & .19 & .666 & 1.20 & .278 & 3.78 & .057 & .26 & .612 \\
\hline \multicolumn{11}{|l|}{ frequency of label use } \\
\hline Nutrient valence $x$ & 1.94 & .168 & .003 & .953 & 1.50 & .226 & .48 & .493 & .03 & .866 \\
\hline \multicolumn{11}{|l|}{ eating attitude } \\
\hline Nutrient valence $x$ & 2.03 & .160 & 2.66 & .108 & 1.17 & .284 & .60 & .441 & 1.24 & .269 \\
\hline BMI & & & & & & & & & & \\
\hline
\end{tabular}


INTERPRETATIONS OF NUTRIENT QUANTITIES

Table A2.

F-and p-values in the ANCOVAs for Interpretations of Verbal Labels in Experiment 2

\begin{tabular}{lcccc}
\hline Factor & $\boldsymbol{d} \boldsymbol{f}$ & Error $\boldsymbol{d f}$ & $\boldsymbol{F}$ & $\boldsymbol{p}$ \\
\hline Quantity & 4 & 2,567 & 10.29 & $<.001^{* * *}$ \\
Nutrient valence & 1 & 644 & 6.97 & $.008^{* *}$ \\
Quantity $\times$ nutrient valence & 4 & 2,567 & 2.24 & .112 \\
Frequency of label use & 1 & 644 & .31 & .581 \\
Eating attitude & 1 & 644 & .25 & .617 \\
BMI & 1 & 644 & .31 & .577 \\
Gender & 1 & 644 & 1.78 & .183 \\
Age & 1 & 644 & 1.29 & .258 \\
Education level & 1 & 644 & .891 & .345 \\
Ethnicity & 1 & 644 & 1.66 & .199 \\
Occupation & 1 & 644 & .02 & .889 \\
Native English-speaker & 1 & 644 & .253 & .615 \\
Nutrient valence $\times$ frequency of label use & 1 & 644 & .48 & .487 \\
Nutrient valence $\times$ eating attitude & 1 & 644 & .16 & .691 \\
Nutrient valence $\times$ BMI & 1 & & & \\
\hline
\end{tabular}

